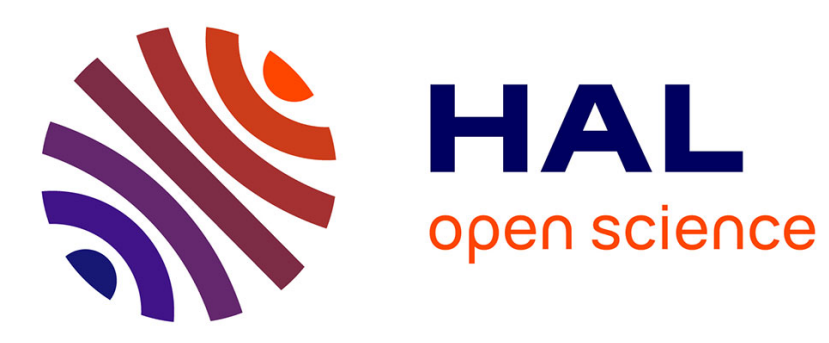

\title{
Optimal Configurations for Perfectly Matched Layers in FDTD Simulations
}

\author{
Xisto Lucas Travassos, Sergio Luciano Avila, D. Prescott, Alain Nicolas,
} Laurent Krähenbühl

\section{- To cite this version:}

Xisto Lucas Travassos, Sergio Luciano Avila, D. Prescott, Alain Nicolas, Laurent Krähenbühl. Optimal Configurations for Perfectly Matched Layers in FDTD Simulations. IEEE Transactions on Magnetics, 2006, 42 (4), pp.563-566. 10.1109/TMAG.2006.871471 . hal-00111254

\section{HAL Id: hal-00111254 \\ https://hal.science/hal-00111254}

Submitted on 4 Nov 2006

HAL is a multi-disciplinary open access archive for the deposit and dissemination of scientific research documents, whether they are published or not. The documents may come from teaching and research institutions in France or abroad, or from public or private research centers.
L'archive ouverte pluridisciplinaire HAL, est destinée au dépôt et à la diffusion de documents scientifiques de niveau recherche, publiés ou non, émanant des établissements d'enseignement et de recherche français ou étrangers, des laboratoires publics ou privés. 


\title{
Optimal Configurations for Perfectly Matched Layers in FDTD Simulations
}

\author{
X. L. Travassos, Jr. ${ }^{1}$, S. L. Avila ${ }^{1}$, D. Prescott ${ }^{2}$, A. Nicolas ${ }^{1}$, and L. Krähenbühl ${ }^{1}$ \\ ${ }^{1}$ Centre de Génie Electrique de Lyon (CEGELY), Laboratoire CNRS-UMR 5005, Ecole Centrale Lyon, Ecully 69130, France \\ ${ }^{2}$ Defense Science and Technology Organization, Edinburgh SA 5111, Australia
}

\begin{abstract}
To be effective a perfectly matched layer (PML) must be reflectionless to all impinging waves over a broad band of frequencies whilst not overly increasing the computational burden. This can be difficult to achieve as one must find a balance between the discretization error, the number of PML layers and the reflection coefficient. This work presents an optimized solution for simulating PMLs for the finite-difference time-domain (FDTD) technique using both the mono-objective genetic algorithm (GA) and multiobjective genetic algorithm (MGA). The main goal is to implement a tool capable of finding the best choice of parameters for the conductivity profile to achieve a minimum for both the reflection and computation time.
\end{abstract}

Index Terms—Error estimation, genetic algorithm (GA), numerical techniques, optimization.

\section{INTRODUCTION}

$\mathbf{T}$ HE perfectly matched layer (PML) is realized from the physical absorption of the incident numerical wave by means of a lossy medium. This is devised using a novel split-field formulation of Maxwell's equations where each vector field component is split into two orthogonal components [1]. Unfortunately the PML requires a considerable amount of computational storage in contrast with analytical boundary conditions due to the storage of split-fields and the number of layers used to truncate the domain of study.

Reflections from the PML exist due to the finite-difference time-domain (FDTD) discretization of the conductivity profile. Recent efforts have been made to reduce these reflections: in [2] a two-step conductivity profile was implemented using a microgenetic algorithm (GA) to obtain the optimal values for each sub-layer, and in [3], a steady-state GA was used to optimize the conductivity profile by minimizing a function of the local error of a two-dimensional (2-D) FDTD code (both are monoobjective optimization).

In this paper, we present an alternative way to design PML boundaries that fulfill the requirements of realistic projects. This optimization process is composed of two stochastic procedures. First, a multiobjective genetic algorithm (MGA) is used to find a solution for the size of a PML which minimizes the reflection coefficient and the computational cost. Next, a GA is used to find a better conductivity profile in order to further reduce the reflectivity. A theoretical approach is utilized for the calculation of the reflection coefficient for a PML [4].

\section{REFLECTION FROM THE PML BOUNDARY}

A numerical method was developed in [4] which computes an exact prediction of the reflection from PML absorbing layers without performing an FDTD simulation. This method considers the two mechanisms which govern the amount of reflection created by a PML boundary. These are: 1) reflections

Digital Object Identifier 10.1109/TMAG.2006.871471

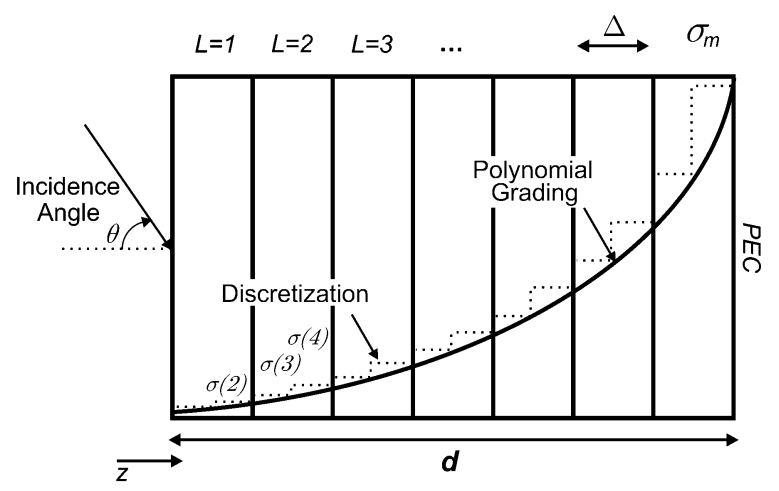

Fig. 1. Schematic of a metal-backed PML.

between PML/free-space and PML/PML interfaces, and 2) the amount of decay experienced as the incident wave travels through the PML medium [4].

The variables necessary to obtain the reflection from the PML are: the normalized wavelength $\lambda / \Delta$, the number of absorbing layers $L$, the incident angle $\theta$, and the conductivity $\sigma$ which is gradually increased from zero to a maximum $\sigma_{m}$ at the perfect electric-wall (Fig. 1) to avoid an abrupt transition between the discrete PML space and the discrete FDTD space. Several profiles have been suggested for grading $\sigma$. The most successful use a polynomial or geometric variation of PML loss with depth $z$. The spatial scale of the conductivity profile using a polynomial grading is given by

$$
\sigma(z)=\sigma_{m}\left(\frac{z}{d}\right)^{n}
$$

where $z$ is the depth within the PML region of total depth $d$, and $n$ is the order of the conductivity's increase.

The design of an optimal PML is made difficult by the requirement to balance the level of reflection against the discretization error in using the variables $\lambda / \Delta, L, \sigma_{m}$ and for a polynomial grading $n$. A large $n$ yields a conductivity distribution that is relatively flat near the PML surface. However, deeper within the PML, $\sigma(z)$ increases more rapidly than for 
small $n$. In this region, the field amplitudes are substantially decayed and reflections due to the discretization error contribute less [5].

However, if $\sigma_{m}$ is small, reflections from the perfect electric conductor (PEC) external boundary will dominate. If $\sigma_{m}$ is large, reflections will be created by the abrupt changes in the inter-layers conductivities (discretization error). If the size of the PML is increased to lessen the inter-layers transition, the computational time and memory requirement will be increased. To understand this balance, we use the MGA in order to facilitate the choice of the best variables.

\section{Genetic Algorithms}

In most optimization problems several goals must be satisfied simultaneously in order to obtain an optimal solution. As these objectives are usually conflicting, no single solution may exist that is best regarding all considered goals. This is what happens in PML design. We recognize that to reduce the reflection coefficient the computational effort must increase. It is important to know this balance to make an efficient FDTD model with low computational cost. Such a scenario requires a multiobjective optimization engine.

Multiobjective optimization seeks to optimize the components of a vector-valued cost function. Unlike single objective optimization, the solution to this problem is not a single point, but a family of efficient points called Pareto optimal front (POF). Each point on this surface is optimal in the sense that no improvement can be achieved in a cost vector component that does not lead to degradation in at least one of the remaining components. Each element in the efficient set constitutes a nondominated (noninferior or nonsuperior) solution to the multiobjective problem [6].

The main goal of the MGA is to find the efficient group of solutions. With this set of solutions it is possible to understand the dependence between each objective, which allows making efficient choices for the final solution decision. The MGA is derived from the GA, which is a stochastic procedure based on the concepts of natural selection in genetics [7]. Basically, the algorithm starts with a set of feasible solutions called the population. The solutions provided by the population are used to form a new population. This is motivated by the hope that the new population will be better than the old one. The new solutions generated are selected according to their fitness (evaluation of objectives). This is repeated until some condition is satisfied, for example, the number of generations.

The GA and MGA used in this work are described in [8].

\section{PML OPTIMIZATION}

Theoretically, the PML interface is reflectionless in the continuum space [9]; this is not the case in the discretized space where there is local step-discontinuity of the conductivity. Since all numerical methods are carried out in the discretized space, one must exercise care to reduce the unwanted reflection due to the finite spatial sampling. A straightforward approach to reducing this unwanted reflection is to make the PML parameters change smoothly along the PML thickness so that the change from grid to grid is small. However, to do this we have to reduce the size of the discretized grid which leads to an increase of the number of unknowns and the computational burden.

The design of an effective PML requires balancing the reflection error, the numerical discretization error and the computational cost associated to the number of the PML layers. Therefore, there is an issue of optimization in determining the PML parameters for best performance.

To address the issue of optimization, we considered the problem where a plane wave is incident on a metal-backed PML obliquely at an angle $\theta$ with the $z$-axis. The goal is to design a PML with the minimal thickness (or minimal computational cost) and maximal absorption quality (or minimal reflectivity for a given range of incidence angles) that are conflicting objectives and hence becomes a multiobjective optimization problem.

The MGA are then coded to find multiple nondominated solutions (the Pareto-front) using a fixed conductivity profile given by (1). The PML parameters to adjust are

$$
P^{g}=\left[\begin{array}{ccc}
L^{g, 1} & n^{g, 1} & \sigma_{m}^{g, 1} \\
\vdots & \vdots & \vdots \\
L^{g, n p} & n^{g, n p} & \sigma_{m}^{g, n p}
\end{array}\right]
$$

where each line represents a feasible solution, $g$ is the current generation and $n p$ is the population size.

The variables to be optimized are then the order of the conductivity increase, the maximal conductivity and the number of layers. They are adjusted to minimize the reflection coefficient for an incident wave range. This becomes the first objective function. The second objective function is to minimize the approximate operations associated to a domain surrounded by $L$ PML layers.

In addition to the MGA procedure, we introduced a GA investigation to improve the conductivity profile given by (1). This investigation used some samples of the POF (set of best solutions found). The conductivity value of each layer is adjusted from a set of feasible solutions determined by a variation between $\pm 10 \%$ of the value found by the MGA.

\section{NUMERICAL RESUlTS}

In order to find the best configuration for the PML absorbing layers we implemented the MGA to work with two goals: to minimize the reflection coefficient and to minimize the computational cost associated with the number of layers [10]. For an $M \times N$ computational space, and assuming $\Delta x=\Delta z$, the approximate operations (AO) count is given by

$$
\mathrm{AO}=20 M N-9(M-2 L)(N-2 L) .
$$

The parameters to be adjusted by the MGA are the variables necessary to obtain the reflection from the PML: the number of PML layers $L$ [4 to 24], the order $n$ of the conductivity profile (1) [0 to 10$]$, the maximum normalized magnetic conductivity $\sigma_{m}^{*} \times \Delta z$ [0 to 1e8]. A computational domain of $M=N=100$ cells was assumed with the normalized wavelength $\lambda / \Delta=20$. The reason for this normalization is that the spatial discretization size can then be neglected as a factor which may affect the 
TABLE I

SOLUTIONS FOUND BY THE MGA FOR $0^{\circ}-50^{\circ}$ ANGLE ABSORPTION

\begin{tabular}{ccccc}
\hline$L$ & $\sigma_{m}{ }^{*} \times \Delta z$ & $n$ & $|R|_{\max }$ & $A O$ \\
\hline \hline 8 & 2634.629 & 3.812 & $2.94 \mathrm{e}-5$ & 136496 \\
10 & 2375.880 & 4.273 & $2.51 \mathrm{e}-6$ & 142400 \\
12 & 2184.965 & 4.266 & $5.30 \mathrm{e}-7$ & 148016 \\
14 & 2298.271 & 4.972 & $1.06 \mathrm{e}-7$ & 153344 \\
16 & 2525.573 & 5.342 & $1.96 \mathrm{e}-8$ & 158384 \\
\hline
\end{tabular}

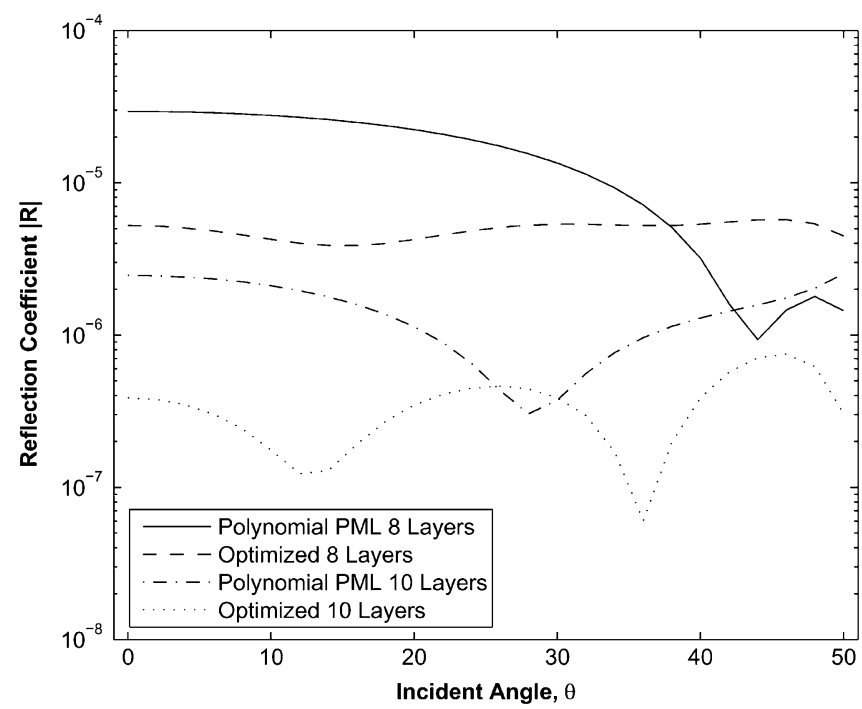

Fig. 2. Reflection coefficient of an optimized PML for $\theta$ from $0^{\circ}$ to $50^{\circ}$.

amount of reflection from a PML boundary. It is also useful to use the form, as this is also used as a criterion by which the FDTD-mesh discretization sizes are chosen [4].

The objective of the optimization procedure was to find a PML with the least reflection over a range of incident angles, $\theta$ from $\theta_{1}$ to $\theta_{2}$.

In this first procedure, efficient solutions are obtained using a population of 100; a crossover probability of 0.9 and a mutation probability of 0.025 . Real-coded MGA are run for 50 generations. Several MGA executions had been carried out to guarantee the POF found. Some samples of the POF are shown in Table I for $\theta_{1}=0^{\circ}$ and $\theta_{2}=50^{\circ}$ at $2^{\circ}$ increments.

Considering the simple polynomial grading of (1), it is clear that optimal values for the polynomial order and the maximum conductivity can be found to achieve suitable solutions with a reasonable number of layers (Table I).

In order to further improve these results, we decided to find an alternative conductivity profile based upon the results of the MGA analysis. To do this we selected some solutions found by the MGA and we performed a process of evolution of the conductivity profile.

Fig. 2 shows the results for $L=[8$ and 10] where it can be seen that for the same number of layers the maximum reflection coefficient in the range is improved by modifying the behavior of the conductivity scaling.

The objective function minimized corresponded to the maximal reflection coefficient in the specified angle range. The convergence has been attained in about 100 generations with a population of 50 individuals in several GA executions.
TABLE II

OPTIMIZED TEN-LAYER CONDUCTIVITY PROFILE FOR $0^{\circ}-50^{\circ}$ ANGLE ABSORPTION

\begin{tabular}{c|c|c|c}
$\sigma_{1}{ }^{*}=0.006$ & $\sigma_{2}{ }^{*}=0.13$ & $\sigma_{3}{ }^{*}=0.715$ & $\sigma_{4}{ }^{*}=2.41$ \\
\hline$\sigma_{5}{ }^{*}=6.218$ & $\sigma_{6}{ }^{*}=13.53$ & $\sigma_{7}{ }^{*}=26.16$ & $\sigma_{8}{ }^{*}=46.32$ \\
\hline$\sigma_{9}{ }^{*}=76.67$ & $\sigma_{10}{ }^{*}=120.27$ & $\sigma_{11}{ }^{*}=180.6$ & $\sigma_{12}{ }^{*}=261.9$ \\
\hline$\sigma_{13}{ }^{*}=368.6$ & $\sigma_{14}{ }^{*}=506.2$ & $\sigma_{15}{ }^{*}=680.1$ & $\sigma_{16}{ }^{*}=895.0$ \\
\hline$\sigma_{17}{ }^{*}=1156$ & $\sigma_{18}{ }^{*}=1482$ & $\sigma_{19}{ }^{*}=1914$ &
\end{tabular}

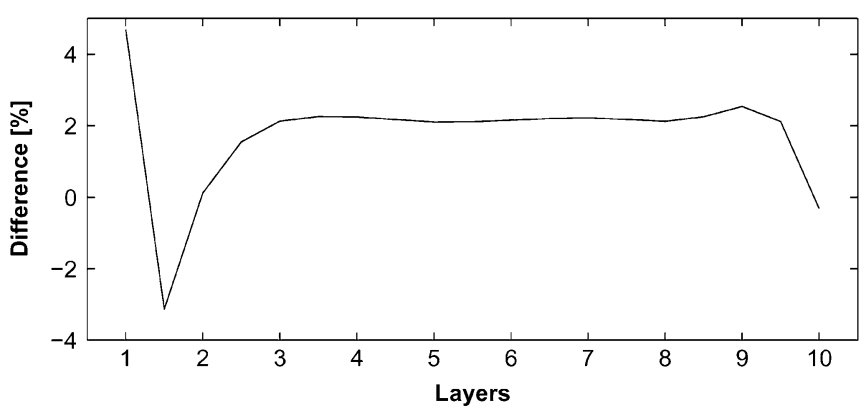

Fig. 3. Difference between the conductivity profile given by (1) and the optimized solution for $L=10$.

The crossover and mutation probabilities were set to 0.9 and 0.05 , respectively. Each generation took about $20 \mathrm{~s}$ with a PC Pentium IV at $1 \mathrm{GHz}$. The entire optimization process took about 45 minutes considering the limits described above.

Table II and Fig. 3 present the conductivity profile and the difference between the polynomial grading of (1) and the new profile found by the GA approach. The FDTD algorithm computes the electromagnetic fields at positions in space which are either on the boundaries of mesh cells or half way between. Hence for a PML of depth $L$ there are actually $2 \times L-1$ (the latest is a PEC wall) different conductivity values.

As shown in Fig. 3 the GA procedure produces subtle modifications throughout the PML absorber. Interestingly, for the majority of cells within the PML where reflections due to the discretization error contribute less, these modifications are all of a very similar magnitude. These changes to the conductivities lead to improvements of almost $16 \mathrm{~dB}$ for the maximal reflection error.

Fig. 4 shows the reflection from the optimized PML constructions as a function of the normalized wavelength. The observation that one can make is that the optmization procedure improved the reflection within a range of around $5 \mathrm{~dB}$ across most of the spectrum.

Table III shows the Pareto-front obtained for the case when $\theta_{1}=40^{\circ}$ and $\theta_{2}=70^{\circ}$. With this scenario, the optimization process became more complex due to the proximity of the tangential incidence to the PML. The MGA procedure experienced some difficulty in finding an improved parameter set due to the reflection coefficient found for $70^{\circ}$. In this case the largest discretization error is manifested at $z=0$, the PML surface. The results for the conductivity profile (1) and the optimized profile for this range are shown in Fig. 5.

Table IV shows the optimized profile for 10 layers. Note how much different these values are to those obtained for a PML designed to absorb across the $0^{\circ}-50^{\circ}$ range (Table II). 


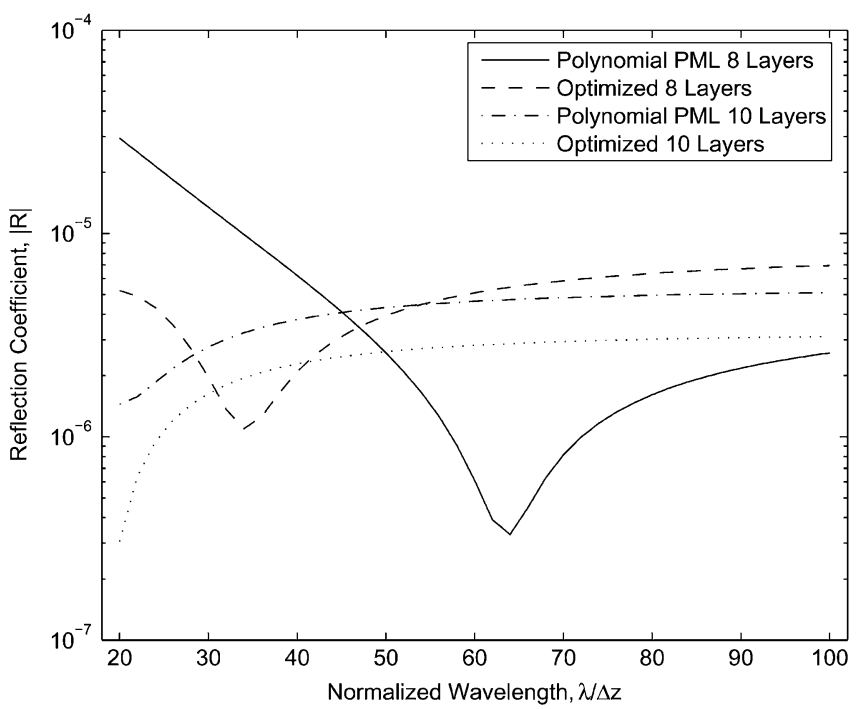

Fig. 4. Reflection from the optimized PML constructions as a function of normalized wavelength with $\theta=0^{\circ}$.

TABLE III

SOLUTIONS Found BY THE MGA FOR $40^{\circ}-70^{\circ}$ ANGLE ABSORPTION

\begin{tabular}{cccc}
\hline$L$ & $\sigma_{m}{ }^{*} \times \Delta z$ & $n$ & $|R|_{\max }$ \\
\hline \hline 8 & 3874.563 & 3.779 & $2.91 \mathrm{e}-5$ \\
10 & 3866.261 & 3.952 & $3.36 \mathrm{e}-6$ \\
12 & 4337.102 & 4.617 & $6.09 \mathrm{e}-7$ \\
14 & 4433.562 & 4.961 & $1.14 \mathrm{e}-7$ \\
16 & 4408.566 & 5.388 & $3.21 \mathrm{e}-8$ \\
\hline
\end{tabular}

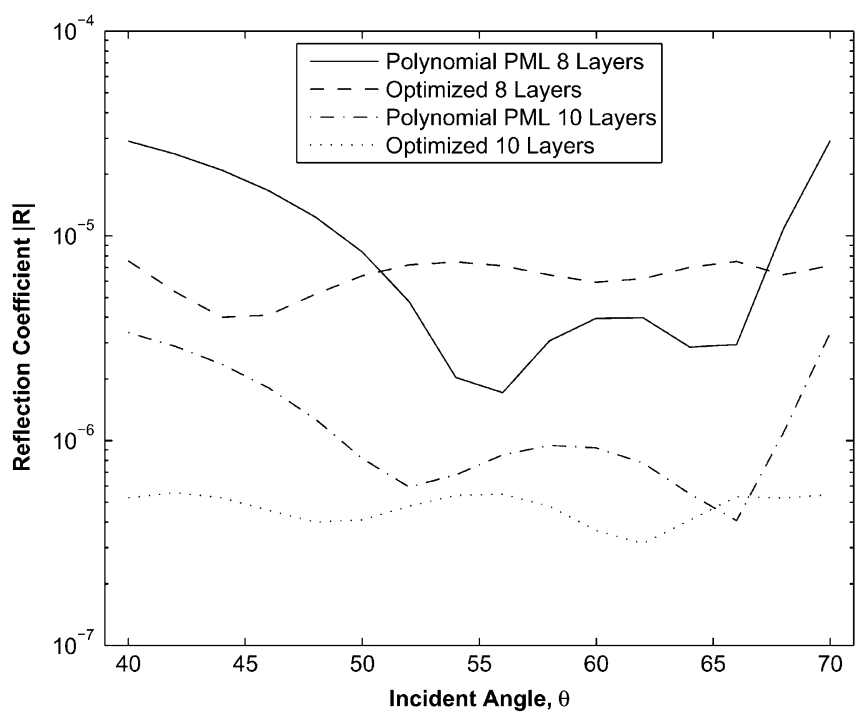

Fig. 5. Reflection coefficient of an optimized PML for $\theta$ from $40^{\circ}$ to $70^{\circ}$.

The results of the optimizations for other layer combinations are tabulated in Table V. It is evident that when $L$ is small the gains achieved through the use of this procedure are much greater than when the number of layers is large. This is attributed to the fact that the GA procedure improves the transition between adjoining conductivity levels. For thin PMLs the relative change between these levels is greater than for the thicker PMLs, hence optimizing these has a greater impact.

In summary, these results show that Berenger's split-field PML can achieve better wide-angle performances in using multiobjective genetic algorithms to find better design parameters.
TABLE IV

OPTIMIZED TEN-LAYER CONDUCTIVITY PROFILE FOR $40^{\circ}-70^{\circ}$ ANGLE ABSORPTION

\begin{tabular}{c|c|c|c}
$\sigma_{1}{ }^{*}=0.028$ & $\sigma_{2}{ }^{*}=0.441$ & $\sigma_{3}{ }^{*}=2.178$ & $\sigma_{4}{ }^{*}=6.778$ \\
\hline$\sigma_{5}{ }^{*}=16.42$ & $\sigma_{6}{ }^{*}=33.73$ & $\sigma_{7}{ }^{*}=62.01$ & $\sigma_{8}{ }^{*}=105.1$ \\
\hline$\sigma_{9}{ }^{*}=167.6$ & $\sigma_{10}{ }^{*}=254.1$ & $\sigma_{11}{ }^{*}=370.2$ & $\sigma_{12}{ }^{*}=521.6$ \\
\hline$\sigma_{13}{ }^{*}=715.2$ & $\sigma_{14}{ }^{*}=959.4$ & $\sigma_{15}{ }^{*}=1261$ & $\sigma_{16}{ }^{*}=1627$ \\
\hline$\sigma_{17}{ }^{*}=2075$ & $\sigma_{18}{ }^{*}=2615$ & $\sigma_{19}{ }^{*}=3184$ &
\end{tabular}

TABLE V

Results of THE OPTIMIZED PROFILE FOR $40^{\circ}-70^{\circ}$ ANGLE ABSORPTION

\begin{tabular}{ccc}
\hline$L$ & $\operatorname{Gain}(d B)$ & $|R|_{\max }$ \\
\hline \hline 8 & 11.73 & $7.54 \mathrm{e}-6$ \\
10 & 15.62 & $5.50 \mathrm{e}-7$ \\
12 & 6.96 & $2.70 \mathrm{e}-7$ \\
14 & 4.36 & $6.93 \mathrm{e}-8$ \\
16 & 2.19 & $2.57 \mathrm{e}-8$ \\
\hline
\end{tabular}

In addition, the optimized PML requires less memory for a desired reflectivity compared with the common design procedure.

\section{CONCLUSION}

This paper demonstrated the application of the GA and MGA procedures in conjunction with a theoretical approach for computing the reflection coefficient to find an improved design configuration for a PML boundary layer. The objectives that were achieved were the minimization of the reflection coefficient and the computational cost of the electromagnetic analysis. This approach removes the burden of seeking adequate $\mathrm{ABC}$ absorption from the FDTD user.

Finally, it was demonstrated that the range of incident angles for which the polynomial-type PML boundaries are most effective could be maximized by optimizing the conductivity grading.

\section{REFERENCES}

[1] J. P. Berenger, "A perfectly matched layer for the FDTD solution of wave-structure interaction problems," IEEE Trans. Antennas Propag., vol. 51, pp. 110-117, 1996.

[2] S. Kim and J. Choi, "Optimal design of PML absorbing boundary condition for improving wide-angle reflection performance," Electron. Lett., vol. 40, pp. 104-106, 2004.

[3] C. L. Li et al., "Optimization of PML absorber's conductivity profile using FDTD," Microw. Opt. Technol. Lett., vol. 37, pp. 380-383, 2003.

[4] D. T. Prescott et al., "Reflection analysis of FDTD boundary conditions Part II: Berenger's PML absorbing layers," Microw. Theory Tech., vol. 45, pp. 1171-1178, 1997.

[5] A. Taflove and S. C. Hagness, Computational Electromagnetics The Finite-Difference Time-Domain Method. Boston, MA: Artech House, 2000.

[6] K. Deb, Multi-Objective Optimization Using Evolutionary Algorithms. New York: Wiley, 2002.

[7] D. E. Goldberg, Genetic Algorithms in Search, Optimization and Machine Learning. New York: Addison Wesley, 1989.

[8] S. L. Avila et al., "An educational tool for teaching optimization in engineering," in Proc. 15TH Compumag Conf., 2005, pp. 216-217.

[9] W. C. Chew and J. M. Jin, "Perfectly matched layers in the discretized space: An analysis and optimization," Electromagn., vol. 16, pp. 325-340, 1996.

[10] J. Schneider and O. M. Ramahi, "A comparison and evaluation of the PML and COM mesh truncation techniques for FDTD simulation," IEEE Trans. Antennas Propag., vol. 3, pp. 1904-1907, 1997.

Manuscript received June 20, 2005; revised November 18, 2005 (e-mail: lucas.travassos@ec-lyon.fr; lucas.travassos@gmail.com). 